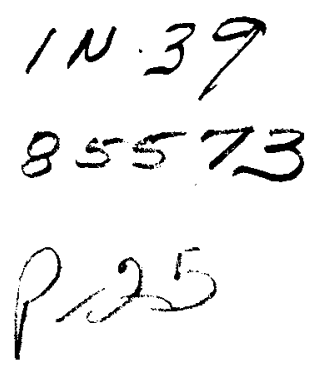

\title{
Macrocrack Interaction With Transverse Array of Microcracks
}

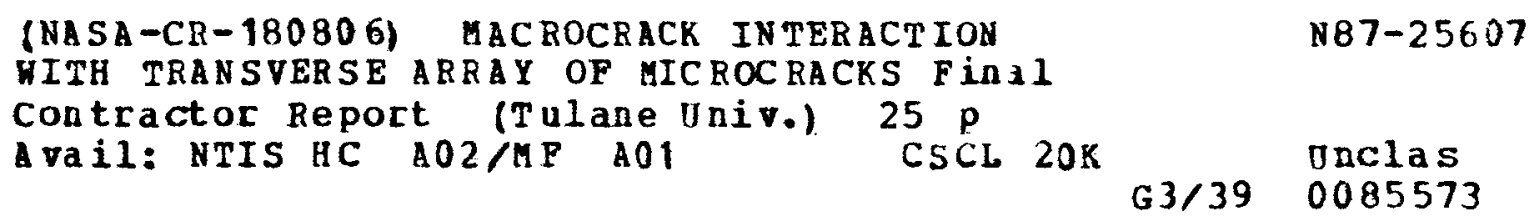

\begin{abstract}
A.A. Rubinstein
Tulane University

New Orleans, Louisiana

and

H.C. Choi

University of Illinois at Urbana-Champaign

Urbana, Illinois
\end{abstract}

July 1987

Prepared for

Lewis Research Center

Under Grant NAG 3-751 


\section{Abstract}

General formulation of a problem involving a macrocrack propagating through an area with microcracks is considered. The applied approach is based on the simultaneous solution of a system of singular integral equations.

Several methods described in the literature are discussed in detail and compared with our approach.

The specific problem considered represents a macrocrack approaching an infinite transverse array of microcracks. Results can be used for interpretation of the microcrack-toughening mechanisms.

Results illustrate the different effects due to loading types in materials with transverse microcrack arrays (stacks of microcracks). Numerical results are compared with data recently appearing in 1 iterature and they demonstrate the importance of the accuracy of the numerical scheme employed. Our results differ by a factor in certain cases as compared with data given in [6].

\section{Introduction}

An accurate assessment of the toughness of structural materials usually requires an estimate of the distortion of the applied (macro) stress field due to material microdefects. As a result, the actual parameters controlling the crack propagation may differ significantly from the expected values based on the applied load. The ratio $\mathrm{K}^{\circ} / \mathrm{K}^{\infty}$ of the actual stress intensity factor (SIF) to the value of SIF which is expected without the influence of microdefects can be taken as a main parameter characterizing the influence of the microdefects on material toughness in the framework of the fracture mechanics of 
brittle materials. In ref. [1] the influence of the single defects is investigated.

It is shown in [1] that a primary effect of the change of the local SIF takes place at a relatively short distance from the crack tip of the main crack to the location of the microdefects (distance in the units of the characteristic length of the defect). Additionally, the location of the defect has a principal role in the overall effect. Thus, cracks, voids, and inclusions, with lower stiffness than the main matrix, located ahead of the macrocrack, increase local SIF. The same defects behind the crack front decrease local SIF. Rigid inclusions or inclusions of higher stiffness than the main material will act in the opposite direction. However, when any of these defects is located at about 90 degrees from the crack line, they act in exactly the same manner (even quantitatively): they shield the main crack. The analysis of the interaction of a semi-infinite array of collinear micro-cracks with the macrocrack [2] shows, as previously, a relatively short range of the influence of the array, but the intensity of the interaction is significant as the macrocrack approaches the array.

The aim of this study is an investigation of the interaction of the macrocrack with a transverse array of parallel microcracks (stack). The problem is formulated in terms of the small scale approach, when the applied stress field is characterized by a remote stress intensity factor. The formulation can be applied to any arbitrary crack distribution and it is straightforward. Generally, the problem involving a finite number of cracks can be solved numerically by reducing the system of integral equations to a system 
of linear algebraic equations. The case of an infinite array is analyzed by applying a certain extrapolation technique in order to obtain a complete effect.

Problems of multicrack interactions have been addressed in literature [1-6]. The approach usually is different to a degree, but the basic principles are of course the same. The difference in the approach, however, can influence the accuracy of the numerical results, assuming a similar computational effort in each case. This can be demonstrated in the following example. Consider the formulation given in [3]. The problem of multiple crack interaction in the infinite plate is posed as a superposition of a single crack homogeneous problems. The traction of each crack includes the terms corresponding to the remote loading and terms called "pseudo-traction." The pseudo-traction components represent the additional traction on each crack due to the crack interactions. Thus, expanding these unknown components in power series by powers of normalized local coordinates, the authors reduce the problem to a system of linear algebraic equations. The number of terms in the expansion dominate the accuracy of the procedure. However, it is easy to show that the error of this calculation will strongly depend on the relative distance between the cracks. The straightforward way to do this would be to consider the case of $\mathrm{N}$ collinear cracks in an infinite plane under remote uniform loading. The resulting stress potentials as per [3] will be

$$
\Phi_{1}^{\prime}(z)=\sum_{i=1}^{N} \frac{1}{2 \pi i} \frac{1}{\left(z-a_{i}\right)\left(z-c_{i}\right)} \int^{c_{i}} \frac{\sqrt{\left(t-a_{i}\right)\left(t-c_{i}\right)}}{t-z} \sigma^{i}(t) d t+\frac{1}{4} \sigma^{\infty}
$$


where $a_{i}, c_{i}$ are coordinates of the $i-t h$ crack tips and $\sigma^{i}$ stands for the total complex surface traction on $i-t h$ crack, including the pseudo-traction, $\sigma^{i}=\sigma_{22} i+i \sigma_{12}-\sigma_{\infty}$. On the other hand, the exact solution can be obtained, and the stress potential will have the following form

$$
\begin{aligned}
& \phi_{2}^{\prime}(z)=\frac{1}{2 \pi i \sqrt{\prod_{i=1}^{N}\left(z-a_{i}\right)\left(z-c_{i}\right)}} \sum_{k=1}^{N} \int_{a_{k}}^{N} \frac{c_{k} \sqrt{\prod_{i=1}^{N}\left(t-a_{i}\right)\left(t-c_{i}\right)}}{t-z} \sigma^{k}(t) d t \\
& \quad+\frac{1}{4} \sigma^{\infty} .
\end{aligned}
$$

Here $\sigma^{k}$ represents the applied traction on $k-t h$ crack. In the case of remote loading $\sigma^{k}=-\sigma^{\infty}$.

Thus, compare the forms (1) and (2). obviously the "pseudo-traction" represents the expansion of square roots on the intervals outside the branch cuts. However, as the distance between the cracks decreases, the number of terms in the expansion has to be increased in order to maintain the desired accuracy. Naturally the expansion will fail as the cracks will coincide (for a branched ( rack).

In ref. [4] the crack opening displacement is taken as an unknown function in representation of the individual potentials and the superposition principle is used. Basically, the formulation is very close to that described above, although it is done in different terms and in the form applicable to three dimensional problems.

A simple and effective approach based on superposition technique and ideas of self-consistency was introduced in ref. $[5,6]$. However, 
the accuracy of this method has to be examined and it will be discussed below. As illustrated above, in the examples of collinear cracks, a superposition of single crack solutions in the form (1) is not always the best way to solve the problem of the interaction of closely located cracks. The form (2) certainly will give better results in terms of accuracy. The alternative to the form follows from the theory of mixed boundary value problems [8]. It is well known that the same function $\phi_{2}(z)$ can be written as

$$
\Phi_{2}^{\prime}(z)=\sum_{k=1}^{N} \frac{A}{2 \pi i} \int_{a_{k}}^{c_{k}} \frac{b_{k}(t)}{t-z} d t+\frac{1}{4} \sigma_{\infty} .
$$

Here $A$ is a constant $\left(A=(E / 4)\left(1-\nu^{2}\right)\right.$ for the plane strain case) and $b_{k}(t)$ is a dislocation density distribution along the crack $\left(a_{k}, c_{k}\right)$. The form (3) represents the general case; the cracks do not have to be collinear. The corresponding function $\psi^{\prime}(z)$ can be obtained as superposition of dislocations as well.

In the following sections the necessary details of the formulation are given and special cases are considered. Special consideration in this report is given to the analysis on a small scale, meaning that microcracks surround the macrocrack, which is much larger than microcracks and therefore is represented as a semi-infinite crack. Two specific microcrack arrays are analyzed. We consider transverse arrays, stacks of microcracks. Type A corresponds to the case when macrocrack is aligned with one of periodically distributed microcracks, and type B corresponds to the case when macrocrack approaches the array in the middle between the microcracks. 


\section{Formulation}

As mentioned above, our formulation is based on the superposition of dislocation arrays representing cracks. Consider the case of a finite number of microcracks. Introduce the stress potentials [7], $\phi(z)$ and $\psi(z)$, so the stress and displacement fields can be represented as follows

$$
\begin{aligned}
& \sigma_{11}+\sigma_{22}=4 \operatorname{Re} \phi^{\prime}(z), z=x_{1}+i x_{2} \\
& \sigma_{22}-\sigma_{12}+2 i \sigma_{12}=2\left(\bar{z} \phi^{\prime \prime}(z)+\psi^{\prime}(z)\right) \\
& u_{1}+i u_{2}=\frac{1}{2}{ }_{\mu}\left[k \phi(z)-\bar{z} \phi^{\prime}(z)-\psi(z)\right] .
\end{aligned}
$$

here $k=3-4 v$ for the plane strain case and $k=(3-v) /(1+v)$ for the plane stress case. $\quad v$ is the Poisson's ratio and $\mu$ is the shear modulus. The analytic functions $\phi^{\prime}(z)$ and $\psi^{\prime}(z)$ are chosen for the case of $\mathrm{N}$ microcracks as

$$
\left\{\begin{array}{l}
\phi^{\prime} \\
\psi^{\prime}
\end{array}\right\}(z)=\left\{\begin{array}{l}
\phi_{0}^{\prime} \\
\psi_{0}^{\prime}
\end{array}\right\}(z)+\sum_{j=1}^{N}\left\{\begin{array}{l}
\phi_{j}^{\prime} \\
\psi_{j}^{\prime}
\end{array}\right\}
$$

where $\phi^{\prime}{ }_{0}(z)$ and $\psi_{o}^{\prime}(z)$ represent the main crack

$$
\begin{aligned}
& \phi_{0}^{\prime}(z)=\frac{A}{\pi i} \int_{-\infty}^{0} \frac{G_{1}(t)}{z-t} d t \\
& \psi_{0}^{\prime}(z)=-\frac{A}{\pi i} \int_{-\infty}^{0} \frac{\overline{G_{1}(t)}}{z-t}-\frac{\bar{t} G_{1}(t)}{(z-t)^{2}} d t .
\end{aligned}
$$

and functions $\phi_{j}^{\prime}(z)$ and $\psi_{j}^{\prime}(z)$ represent the microcracks 


$$
\begin{aligned}
& \dot{\phi}_{j}(z)=\frac{A l_{j}}{\pi i} \int_{-1}^{1} \frac{g_{j}(s) d s}{z-a_{j}-s \ell_{j} e^{i \theta j}}
\end{aligned}
$$

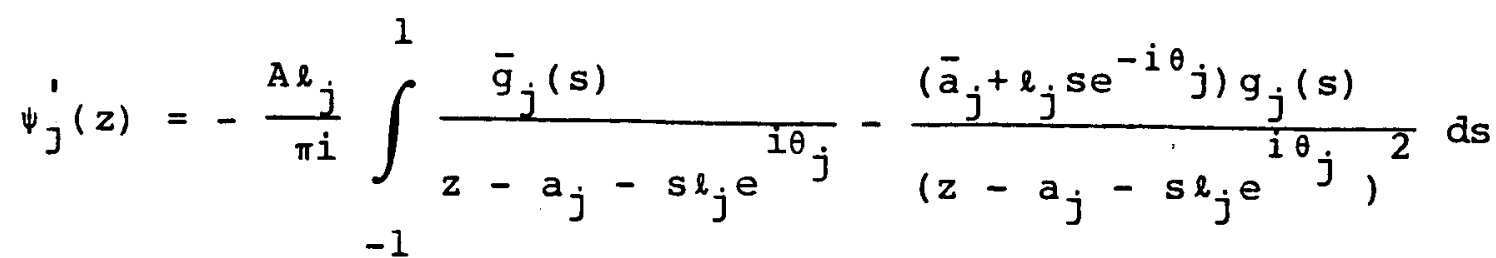

Here $\mathrm{A}=\mu /(\mathrm{k}+1), \mathrm{g}_{\mathrm{j}}(\mathrm{s})$ represents the dislocation density distribution along the microcracks and $G_{I}(t)$ is the dislocation density along the macrocrack. The notations along the microcracks are illustrated in Fig. 1.

The traction free conditions on the crack surfaces lead to a system of singular integral equations for determination of the unknown dislocation density functions. These conditions are

$$
\begin{gathered}
\sigma_{11}+i \sigma_{12}=\phi^{\prime}(z)+\overline{\phi^{\prime}(z)}+\bar{z} \phi^{\prime \prime}(z)+\psi^{\prime}(z)=0 \\
\text { on } x_{i}<0, x_{2}=0
\end{gathered}
$$

and

$$
\sigma_{n n}+i \sigma_{n s}=\phi^{\prime}(z)+\overline{\phi^{\prime}(z)}+e^{2 i \theta} j \quad\left[\bar{z}_{\phi^{\prime \prime}}(z)+\psi^{\prime}(z)\right]
$$

on the j-th microcrack. Additionally, the conditions of single valued displacement have to be stated. This condition, as in the case of multiply connected regions, is reduced to close contour integrals. The contours have to be chosen surrounding each microcrack. Thus one will obtain $\mathbf{N}$ additional equations which are reduced to

$$
\int_{-1}^{1} g_{j}(s) d s=0, j=1,2, \ldots, N \text {. }
$$


The homogeneous system of singular integral equations of the first kind obtained above has a family of solutions. The supplementary conditions (10) apply to finite intervals only. For the semi-infinite interval an additional condition has to be stated. As discussed in [1], the singular integral equation of the first kind on a semi-infinite interval requires a stabilizing term; namely, the terms of the order $o\left(t^{-1 / 2}\right)$ as $t \rightarrow \infty$ have to be specified up to a multiple constant as a parameter of the problem.

Physically, for the considered case it corresponds to a description of the remotely applied stress. Thus the dislocation density has to be taken in the form

$$
\begin{gathered}
G_{1}(t)=G(t)-\bar{K}_{\infty} / 2 A \sqrt{2 \pi t} \\
t+-\infty
\end{gathered}
$$

and

$$
G(t)=o\left(t^{-1 / 2}\right), t+-\infty
$$

substituting (11) into (6), then

$$
\phi_{0}(z)=\frac{A}{\pi i} \int_{-\infty}^{0} \frac{G(t)}{z-t} d t+\frac{K_{\infty}}{2 \sqrt{2 \pi z}}
$$

and

$$
\psi_{0}^{\prime}(z)=-\frac{A}{\pi i} \int_{-\infty}^{0}\left[\frac{\overline{G(t)}}{z-t}-\frac{\bar{E} G(t)}{(z-t)^{2}}\right] d t+\frac{K_{\infty}}{2 \sqrt{2 \pi z}}-\frac{1}{4} \frac{\bar{K}_{\infty}}{\sqrt{2 \pi z}}
$$

$K_{\infty}$ here is the ramote (applied) stress intensity factor. of course, terms corresponding to a remote stress field do not produce traction 
on the macrocrack surfaces. The resulting system with potentials $\phi_{0}$ and $\psi_{0}$ in the form (13) and (14) with restriction (12) is complete and has a unique solution.

Following the theory of singular integral equations [8] and using the numerical technique based on Gauss-Chebyshev quadrature [9] one represents unknown functions as

$$
G(t)=\alpha(u) \sqrt{\frac{1+u}{1-u}}(-1<u<1)
$$

and

$$
\begin{aligned}
g_{j}(s) & =\frac{\beta_{j}(s)}{\sqrt{1-s^{2}}}(-1<s<1) . \\
j & =1,2 \ldots, N
\end{aligned}
$$

Condition (12) becomes

$$
\alpha(-1)=0
$$

The numerical procedure is based on discretization of the integration interval by the nodes of Chebyshev polynomial of the first kind for the dummy variable and the second kind for the variable on the right hand side. The value of interest here is the ratio of the acting SIF at the macrocrack to a remote SIF.

$$
\mathrm{K}_{\mathrm{O}} / \mathrm{K}_{\infty}=1-\mathrm{i} \alpha(1) \cdot \mathrm{A} / \mathrm{K}_{\infty} \cdot
$$

For the values of $\alpha(u)$ at $u=-1$ and $u=1$ Lagrange formulas for the Chebyshev polynomials were used.

\section{Numerical Results}

The formulation above is different from the previously used methods of solution of crack interaction problems. The difference is not significant in its basis, but more or less in its technical 
aspects. The main feature of the above approach is simultaneous solution of a large system of singular integral equations. There is no influence type function for particular boundary conditions in this formulation; none of the conditions has to be satisfied with preferable accuracy. The numerical accuracy is uniform and is controlled by the number of nodes (or, in other words, by the order of the approximating polynomial) on a particular integration interval. So, $L_{j}$ will correspond to a number of nodes on $j-t h$ microcrack and $L_{0}$ will stand for the number of nodes on the macrocrack. The case $L_{j}=2$ will simplify integrals (7), and using Gauss-Chebyshev integration formula one can obtain a simple algebraic system very similar to one used in $[5,6]$.

\section{i) Collinear Cases}

The test cases for our formulation were the problems of collinear macrocrack-microcrack interaction and macrocrack-semiinfinite array interactions. The first case is solved exactly and the second is solved by using a completely different technique in [2]. The numerical solution for the case of a single crack compares well with the exact solution. For the distance between the macrocrack tip and the closest tip of microcrack higher than 0.021 (in the units of a microcrack length), the error is below $0.3 \%$ with a relatively low number of nodes used in the computations. For higher values of this distance the error is rapidly decreasing. The data of this comparison is given in Table 1. In table 1 , the data corresponding to a semi-infinite microcrack array is given as well. 
The numerical solution of the problem dealing with an infinite number of cracks is simplified by using the effect of the order of microcrack distribution. As a result, we seek the difference between dislocation density distribution functions of the neighboring cracks instead of the function itself. This difference approaches to zero faster than the dislocation density function. Therefore, a number of equations in the system can be reduced. Thus we take in (16)

$$
\beta_{j}(s)=\beta_{1}(s)+\sum_{k=1}^{j} \Delta \beta_{k}(s)
$$

Additionally, solution of the problem on a small scale is associated with decaying remote stress as $1 / z$, so the dislocation density on the cracks located at sufficient distance from the main crack can be expected to decay as

$$
\max \left|B_{k}(s)\right| \simeq \frac{\text { const }}{\sqrt{a_{k}}} \text {, as } a_{k} \rightarrow \infty \text {. }
$$

Incorporation of (19) and (20) into the numerical procedure allows one to reduce the number of equations and at the same time to keep the effect of distant microcracks present. The results given in Table 1 correspond to $N=15$ (number of $\Delta \beta_{k}$ evaluated) but the total number of microcracks taken into account using (20) is up to 200. The results in Table $l$ indicate an excellent consistency of the numerical sequence and indicate mainly the influence of the number of nodes chosen in the procedure.

(ii) Macrocrack interaction with an array of parallel macrocracks. As mentioned above, the numerical technique outlined 
here can be applied to an arbitrary crack distribution. In this report we focus our attention on the cases of parallel microcrack arrays (stacks of microcracks) transverse to a macrocrack. We consider two types of parallel microcrack distributions. The array of type $A$ has a microcrack on the $x$-axis contrary to type B. Both arrays are symmetric with respect to the axis and are equally spaced with a pitch $p$. Thus in both cases $\theta_{k}=0$ and in the case $A$

$$
a_{k}=d+\frac{\ell}{2}+i p k
$$

and in the case $B$

$$
a_{k}=d+\frac{\ell}{2}+i p \frac{k}{2}
$$

in (21) and (22) $k=\ldots,-3,-2,-1,0,1,2,3, \ldots$ The symmetry of the problem reduces the number of unknown functions by using the following relations:

$$
\begin{aligned}
& g_{-k}(s)=-\overline{g_{k}(s)} \text { for Mode I loading and } \\
& g_{-k}(s)=g_{k}(s) \text { for Mode I I loading. }
\end{aligned}
$$

As guiding cases, we consider the cases of three parallel cracks for type $A$ and two cracks for type B. These cases were studied in order to determine the regions of significant influence of the microcrack on the stress intensity factor at the main crack. Thus, we found that at the ratio of $\mathrm{p} / \ell \geq 14$ ( $\mathrm{p}$ here is the distance from the microcrack to $x$ axis) the effect of two microcracks (outside in the case A) is less than $0.1 \%$, regardless of the distance ratio d/ $\ell$. In our calculations, infinite arrays are modeled by arrays of half length $N_{C}=15$ for $P / \ell>1.0$ ( $p$ now is the pitch of the microcrack distribution) and $N_{C}=30$ for $P / \ell<1.0$. Additionally, in order to 
obtain results with uniform accuracy, the number of nodes on each microcrack is chosen in relation to the distance of the array from the macrocrack tip. For example, for the smaller values of $d / \ell, 1$ ike d/ $\ell<0.05$ we used 11 nodes on each microcrack and a smaller number of nodes for the larger ratio $d / l$. The minimum number of nodes used was 7 .

The results for the infinite microcrack array of type $A$ are given in Fig. 2 and 3 , for the loading modes I and I respectively. The corresponding results for the array of type B are given in Fig. 4 and 5. We compare results obtained here for the arrays with the data given for a single crack along the crack line [2] in case A (and B when applicable: $d>0$ ) and the case $B$ is compared with the case of two parallel microcracks equally distant from the crack line.

The main feature of the effect produced by the transverse array is the shielding effect of the remote microcracks. As demonstrated in [7] by analyzing the solution for an arbitrary positioned microcrack in the vicinity of the macrocrack tip, the effect on the resulting SIF depends on the angular position of the microcrack and on its orientation. This data observation is supported by an approximate analysis given in [10]. Microcracks with positional angle higher than $62 \mathrm{deg}$. will produce a shielding effect. Thus for the transverse array there will always be a portion that will act as a shield. The effectiveness of the shielding depends on the ratio d/l. As evident from the presented data, the cracks located closer to the main crack dominate the resulting interaction effect. Practically (within 1-5\% accuracy) only 3-4 first microcracks in each half plane control the overall result. 


\section{Concluding Remarks}

A simple approach based on the solution of a system of integral equations was applied to a problem of a macrocrack quasi-statically propagating through an array of parallel microcracks (stack of microcracks). This analysis was aimed at evaluating an effect of microcracking on overall material toughness. The method and the numerical formulation were carefully examined in cases with known solutions and were found to be highly accurate. As explained in the introduction, the number of nodes taken into the numerical scheme or taken into account for the development of simplified techniques has a significant impact on the final results. For example, our results in some regions are significantly different from the data presented by Kachanov and Montagut [6] in similar geometries. Thus, in the case of a macrocrack approaching two parallel cracks, the maximal shielding effect according to [6] is about 25\%. In our computations the same geometry will yield $77 \%$ shielding. Similarly, cases of the infinite arrays give different results. Evidently, the numerical approach used in [6] will give better results for geometries when cracks do not overlap each other, or spread at larger distances.

The overall results of this study demonstrate the significant effect of microcrack shielding (reduction of the actual value of the stress intensity factor with respect to one remotely applied). Two modes I and II were examined and found to have somewhat different effects. If one can consider such factors as stability of the shielding effect, which would depend on geometrical deviations of the considered configuration, and secondly on the width of the region with qualitatively similar effect. The first factor was not 
considered here but certainly is expected to be related to the second. The second factor can be judged by observing results, and it is evident that mode I gives stable shielding and mode II does not. In the case of mode II we have two regions, shielding and antishielding, very close to each other, and therefore the total shielding effect hardly can be expected in a practical situation. Thus Mode I can be characterized by reduction of the applied stress intensity factor by $80 \%$ which would lead to a material toughness increase of four times.

The Mode II on the other hand can not claim a stable shielding effect. If in the Mode I case the crack advances toward the stack of microcracks will be stimulated by the microcrack up to the point when they overlap with the macrocrack and then stable shielding takes place; in the case of Mode II after similar development, the next region will still stimulate crack growth and so slight deviation of the macrocrack tip from the shielding zone will still stimulate crack growth. This effect can explain different toughness of porous materials when they are subjected to shear stresses. 
[1] A. A. Rubinstein, "Macrocrack-Microdefect Interaction," Journal of Applied Mechanics, V. 53, pp. 505-510 (1986).

[2] A. A. Rubinstein, "Macrocrack Interaction with Semi-Infinite Microcrack Array", Int. J. Fracture, V. 27, pp. 113-119 (1985).

[3] H. Morri and S. Nemat-Nasser, "Estimate of Stress Intensity Factors for Interacting Cracks", Advances in Aerospace Structures, Materials and Dynamics, ed. U. Yugeogle et. al., pp. 111-117 (1983).

[4] A. Chudnovsky, A. Dolgopolsky and M. Kachanov, "Elastic Interaction of a Crack With a Microcrack Array: Part I and Part II", Int. J. Solids and Structures (in press).

[5] M. Kachanov, "A Simple Technique of Stress Analysis in Elastic Solids with Microcracks", Int. J. of Fracture, V. 28, pp. R11-R19 (1985).

[6] M. Kachanov and E. Montagut, "Interaction of a Crack with a Certain Microcrack Arrays", Eng. Fracture Mechanics, Vol. 25, pp. 625-636, (1986).

[7] N. I. Muskhelishvili, Some Basic Problems of the Mathematical Theory of Elasticity, Noordihoff, Groninger (1975).

[8] N. I. Muskhel ishvili, Singular Integral Equations, Noordhoff, Groningen (1953).

[9] F. Erdogun and G. D. Gupta, "On the Numerical solution of Singular Integral Equations", Quarterly of Applied Mathematics, v. 29, pp. 525-534, (1972).

[10] L.R.F. Rose, "Microcrack Interaction With a Main Crack", Int. J. of Fracture, 31, pp. 233-242, (1986). 
Table 1. SIF for a Single Crack and Semi-Infinite Array Distributed Along $x$-axis.

\begin{tabular}{|c|c|c|c|c|c|c|}
\hline & \multicolumn{2}{|c|}{$\mathrm{d}=0.021, \quad \mathrm{P}=1.5$} & \multicolumn{2}{|c|}{$d=0.1, \quad P=2.0$} & \multicolumn{2}{|c|}{$d=0.2, \quad P=5.0$} \\
\hline & $\mathrm{K}_{\mathrm{s}} / \mathrm{K}_{\infty}$ & $\mathrm{K} / \mathrm{K}_{\infty}$ & $\mathrm{K}_{\mathrm{s}} / \mathrm{K}_{\infty}$ & $\mathrm{K} / \mathrm{K}_{\infty}$ & $\overline{K_{s} / K_{\infty}}$ & $\mathrm{K} / \mathrm{K}_{\infty}$ \\
\hline Ref.[2] & 2.14848 & 2.26081 & 1.38738 & 1.42075 & 1.20923 & 1.21237 \\
\hline $\begin{array}{l}\text { Result of } \\
\text { Present } \\
\text { Computations }\end{array}$ & 2.14212 & 2.25618 & 1.3874 & 1.42137 & 1.20922 & 1.21380 \\
\hline Error \% & 0.3 & 0.2 & 0.003 & 0.04 & 0.0008 & 0.1 \\
\hline$L_{0}, L_{J}, N$ & $40,1]$ & 15 & 40 & 15 & 40,8 & 15 \\
\hline
\end{tabular}

$d$ is distance between macrocrack and first crack in the array, $p$ is the pitch of the crack distribution. L is the number of noded on on the macrocrack. L $L_{j}$ is number of nodes on each microcrack and $N$ is the number of microcracks for which $\Delta \beta_{k}(s)$ is evaluated. 


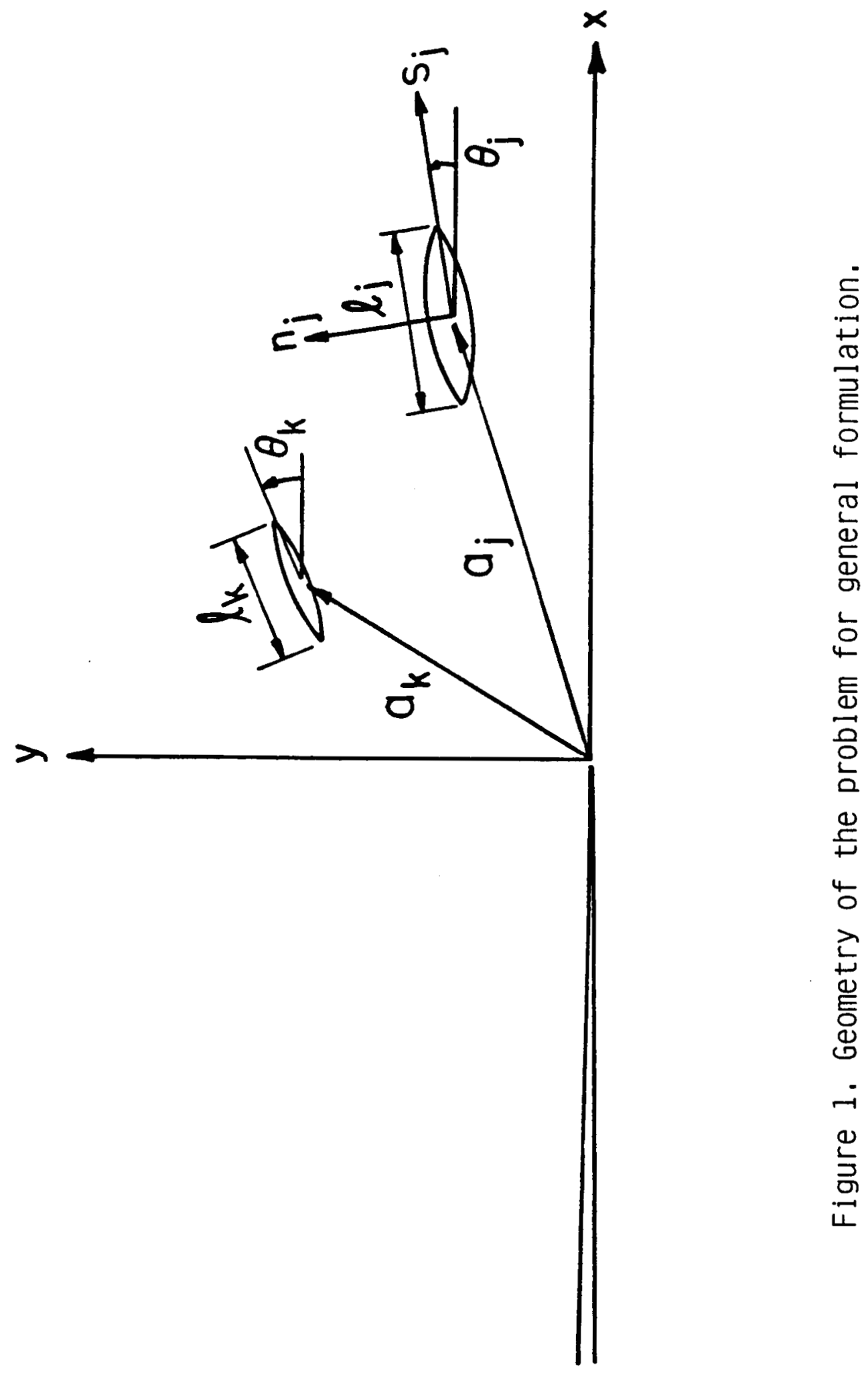




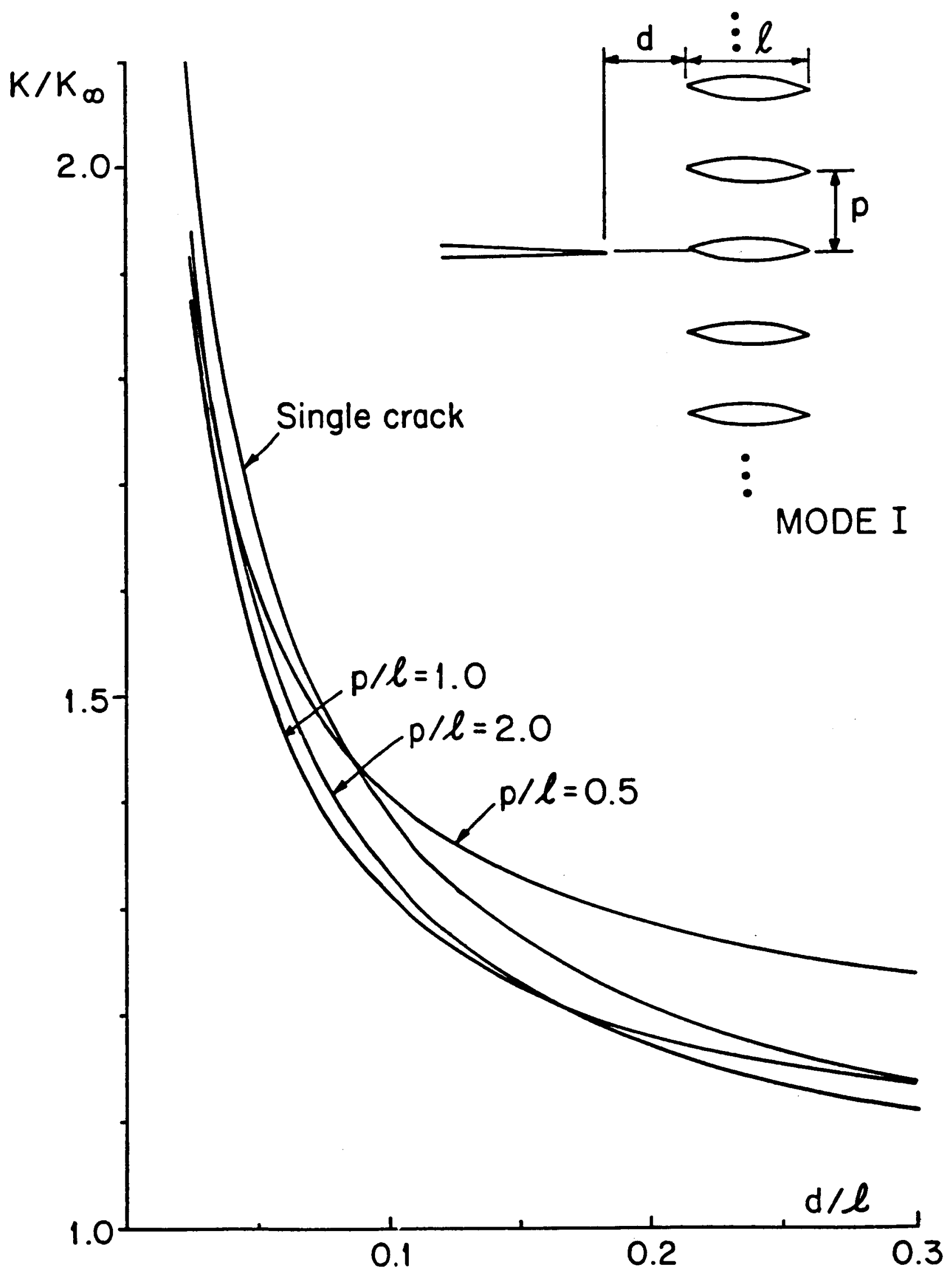

Figure 2. Results of the computation for the case of array A, Mode I. 


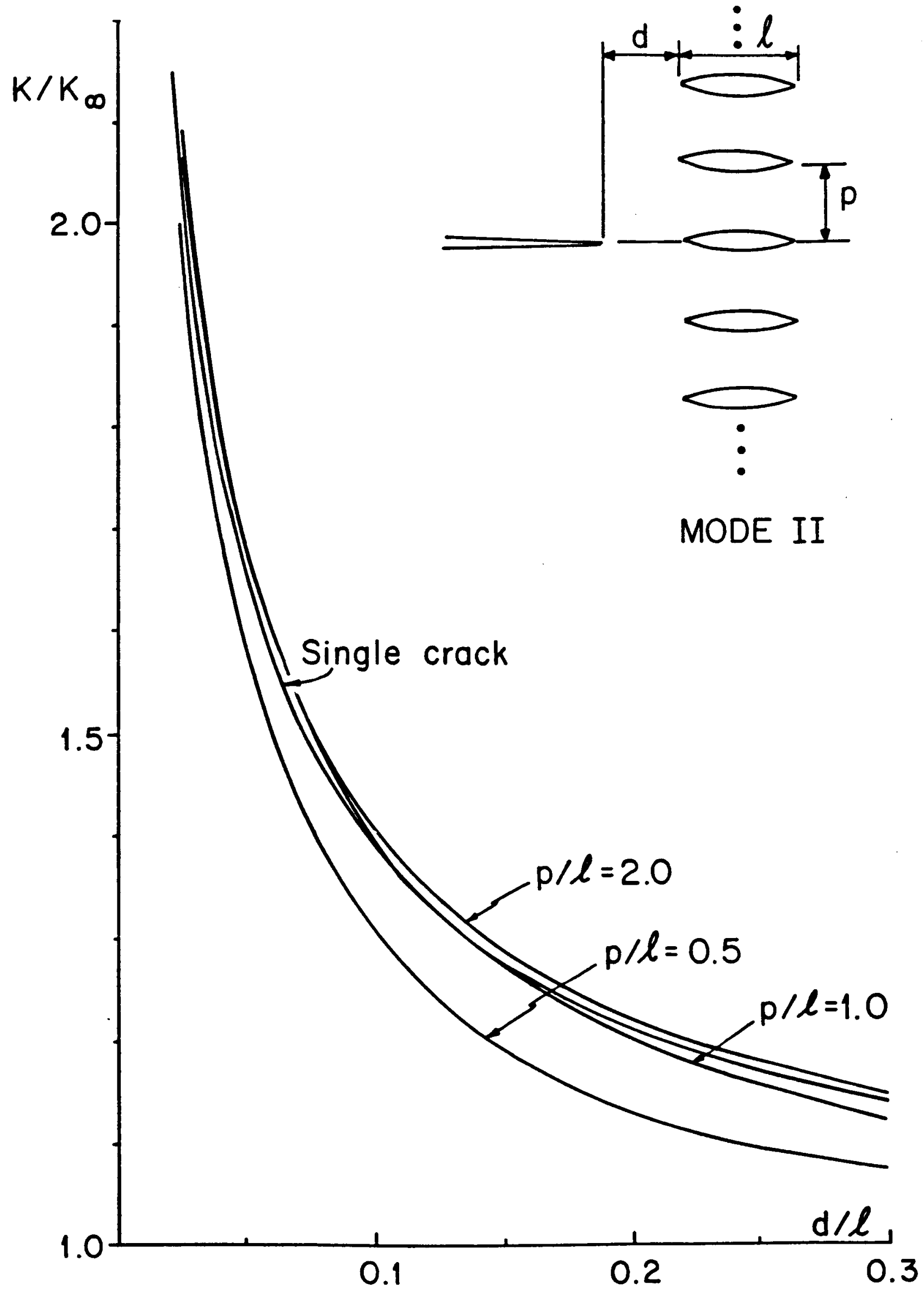

Figure 3. Results of the computation for the case of array A, Mode II. 20 


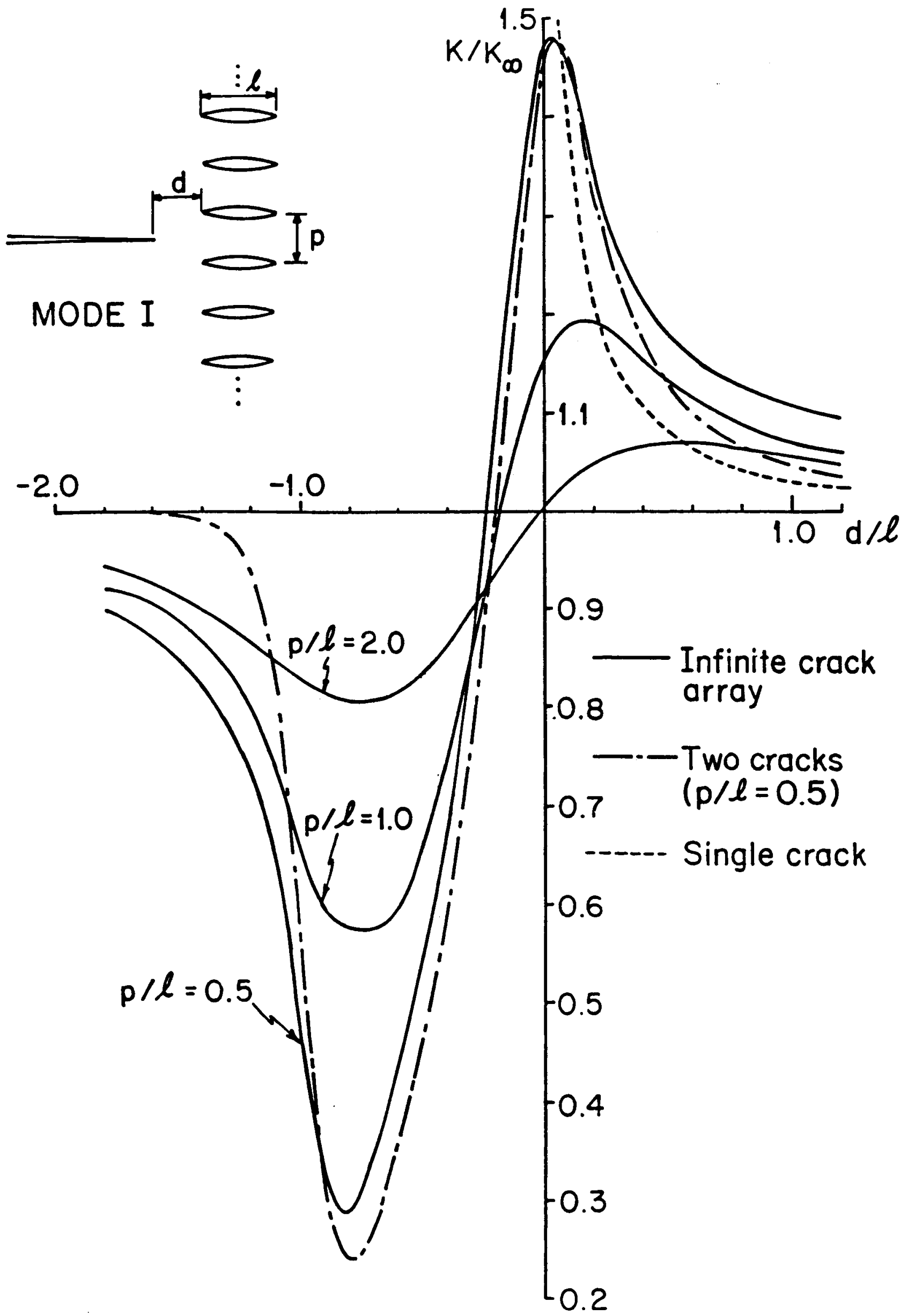

Figure 4. Results of the computation for the case of array B, Mode I. 
MODE II

TYPE $B$

I




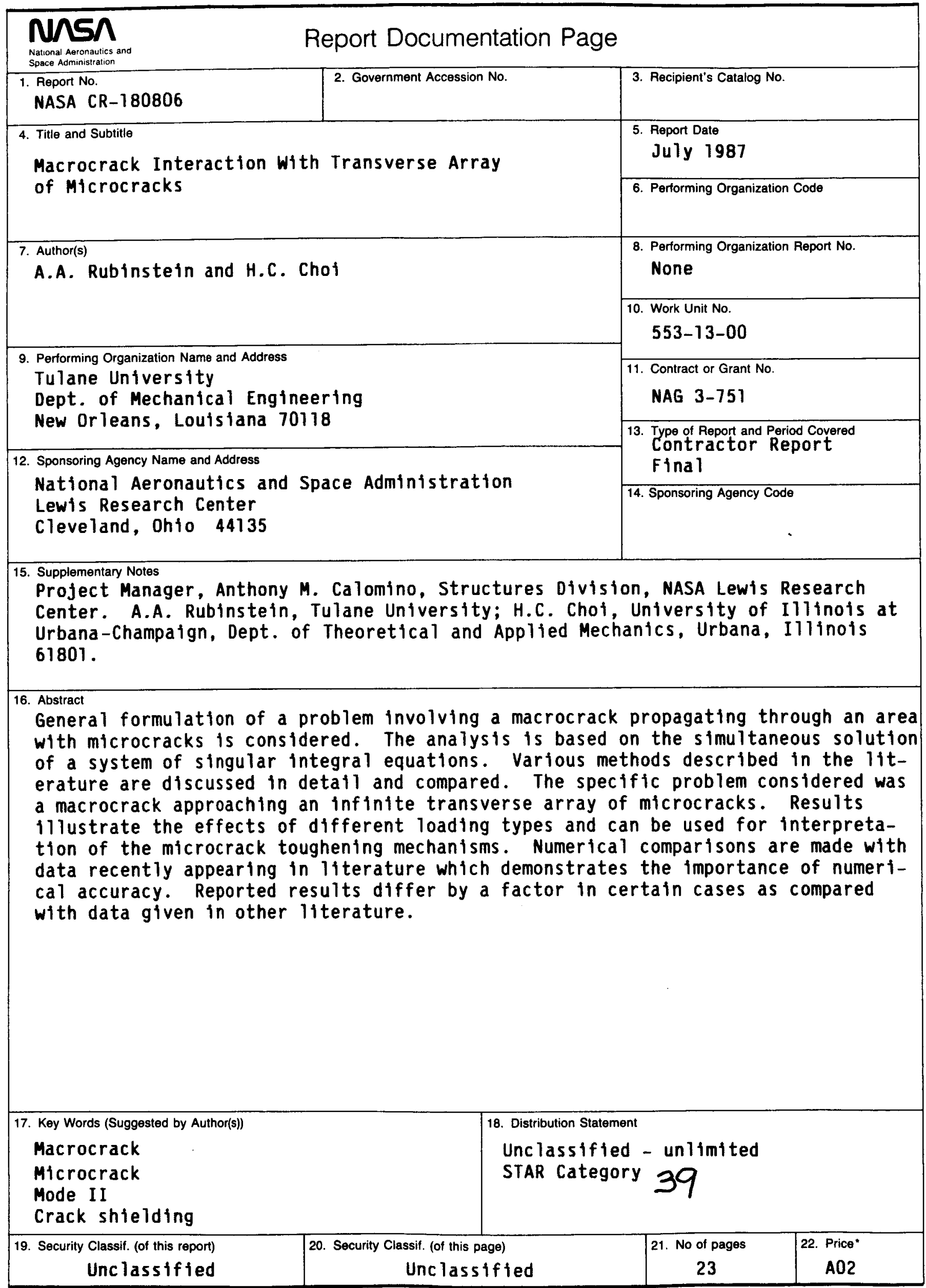

NASA FORM 1626 OCT $86 \quad$ *For sale by the National Technical Information Service, Springfield, Virginia 22161 